\title{
The plant growth regulator methyl jasmonate inhibits aflatoxin production by Aspergillus flavus
}

\author{
Marta Goodrich-Tanrikulu, Noreen E. Mahoney and \\ Susan B. Rodriguez†
}

US Department of Agriculture, Agricultural Research Service, Western Regional Research Center, 800 Buchanan Street, Albany, CA 94710, USA

\author{
Author for correspondence: Marta Goodrich-Tanrikulu. Tel: +1 510559 5627. Fax : +1 5105595777. \\ e-mail: martat@pw.usda.gov
}

\begin{abstract}
Aflatoxins are highly toxic and carcinogenic compounds produced by certain Aspergillus species on agricultural commodities. The presence of fatty acid hydroperoxides, which can form in plant material either preharvest under stress or postharvest under improper storage conditions, correlates with high levels of aflatoxin production. Effects on fungal growth and aflatoxin production are known for only a few of the numerous plant metabolites of fatty acid hydroperoxides. Jasmonic acid (JA), a plant growth regulator, is a metabolite of 13-hydroperoxylinolenic acid, derived from $\alpha$-linolenic acid. The volatile methyl ester of JA, methyl jasmonate (MeJA), is also a plant growth regulator. In this study we report the effect of MeJA on aflatoxin production and growth of Aspergillus flavus. MeJA at concentrations of $10^{-3}-10^{-8} \mathrm{M}$ in the growth medium inhibited aflatoxin production, by as much as $96 \%$. Exposure of cultures to MeJA vapour similarly inhibited aflatoxin production. The amount of aflatoxin produced depended on the timing of the exposure. MeJA treatment also delayed spore germination and inhibited the production of a mycelial pigment. These fungal responses resemble plant jasmonate responses.
\end{abstract}

Keywords: aflatoxin, Aspergillus flavus, Aspergillus parasiticus, lipoxygenase, methyl jasmonate

\section{INTRODUCTION}

Aflatoxins are highly toxic and carcinogenic secondary metabolites produced by the fungi Aspergillus flavus, $A$. parasiticus and $A$. nomius. Aflatoxin contamination of agricultural commodities, including corn, cottonseed, peanuts, and many other oil-rich crops such as tree nuts, can occur following Aspergillus spp. infection (Payne, 1992), and is therefore a public health concern. Aflatoxin levels in crops often rise following exposure to certain stresses such as high temperature and drought (Payne, 1992; Sanders et al., 1993). One factor influencing the production of aflatoxin is the presence of high levels of oxidized fatty acids such as fatty acid hydroperoxides (Fanelli \& Fabbri, 1989), often found under stress (Merzlyak, 1990). Fatty acid hydroperoxides can be formed by autooxidation, or enzymically by lipoxygenases

†Present address: Department of Biological and Physical Sciences, Indiana University, Kokomo, IN 46904, USA.

Abbreviations: JA, jasmonic acid; MeJA, methyl jasmonate. acting on $\alpha$-linoleic and $\alpha$-linolenic acids (Vick, 1993). These hydroperoxides stimulate the formation of aflatoxin by $A$. flavus and $A$. parasiticus (Fabbri et al., 1983; Fanelli \& Fabbri, 1989). Degradation of the hydroperoxides by later steps in the plant lipoxygenase pathway leads to multiple byproducts, depending on the polyunsaturated fatty acid substrate, the positional specificity of the lipoxygenase, and the activities of enzymes catalysing the subsequent steps. The aldehydes hexanal, trans-2-hexenal, and trans-2-nonenal, which are hydroperoxide metabolites via hydroperoxide lyase (Vick, 1993), generally inhibit spore germination (Zeringue \& McCormick, 1990) and aflatoxin production (Zeringue \& McCormick, 1990; Zeringue, 1991; Zeringue \& Bhatnagar, 1994), although stimulation of aflatoxin production has been observed under some conditions (Fabbri et al., 1983; Zeringue, 1991).

Another $\alpha$-linolenic acid metabolite, via lipoxygenase and hydroperoxide dehydratase, is jasmonic acid (JA) (Vick, 1993). JA and closely related compounds, such as its methyl ester, $\mathrm{MeJA}$, are endogenous plant growth 
regulators. Jasmonates appear to be ubiquitous in both higher and lower plants (Staswick, 1992; Sembdner \& Parthier, 1993). The jasmonates regulate a diverse set of responses, including inhibition of pollen and seed germination, altered synthesis of secondary metabolites, suppression of protein synthesis, and the activation of defence responses, in part by altering gene expression (reviewed by Parthier, 1991; Staswick, 1992; Sembdner \& Parthier, 1993). MeJA is more volatile than JA, so exposure to it either in solution or in the gaseous phase can elicit plant responses. In addition, it is used as a fragrance in the perfume industry (Staswick, 1992).

JA was first identified as a plant growth inhibitor when it was isolated from culture filtrate of the fungus Lasiodiplodia (Botryodiplodia) theobromae, a plant pathogen (Aldridge et al., 1971). Reports of direct effects of jasmonates on fungi are limited, and include the inhibition of mycorrhizal fungal growth (Gogala, 1991) and the inhibition of appressoria formation or spore germination in three plant pathogens (Neto et al., 1991; Schweizer et al., 1993, Cohen et al., 1993).

Exposure of aflatoxigenic fungi to jasmonates is likely, since the fungi grow on plant material. Thus, we began this study by examining the effects of MeJA on a toxigenic strain of $A$. flavus growing in vitro. In this experimental system, effects of MeJA can be studied in a defined medium, and are therefore separable from effects of a plant/fungal interaction.

\section{METHODS}

Cultures, media and chemicals. MeJA was a gift from Bedoukian Research. JA, and standards of aflatoxins $B_{1}, B_{2}$ and $\mathrm{G}_{1}$, sterigmatocystin, $O$-methylsterigmatocystin and cyclopiazonic acid were purchased from Sigma.

Spores (approximately 6000 in $5 \mu \mathrm{l}$ suspension) of $A$. flavus strain WRRC 3-90-42-12 (NRRC no. 25347) were inoculated as detailed by Rodriguez \& Mahoney (1994) onto the centre of $60 \mathrm{~mm}$ Petri dishes. Plates contained Vogel's medium N (VMN) (Davis \& deSerres, 1970) or modified CYA (Rodriguez \& Mahoney, 1994) agar, with or without MeJA or JA added after autoclaving. For exposure to levels less than $10^{-4} \mathrm{M}$, dilutions of the MeJA stock were made in ethanol. Cultures were incubated at $25^{\circ} \mathrm{C}$.

Spore germination time was determined in both liquid and solid VMN in the presence or absence of $10^{-4} \mathrm{M}$ MeJA or JA. The percentage of spores with germ tubes was counted microscopically at various times after inoculation. Regression lines were fitted to a plot of the percentage of germinated spores versus time after inoculation. The time required for $50 \%$ germination was used to compare mean germination times among treatments.

For exposure of cultures to airborne MeJA, the MeJA and the fungal inoculum were physically separated by removing a strip of agar ( $3 \mathrm{~mm}$ wide) between them (see Fig. 1). Some experiments tested the effects of shorter exposures to MeJA. In one set of these studies, the MeJA $(0 \cdot 2 \mu \mathrm{l})$ was added across the gap from the growing culture at defined intervals after inoculation. In a complementary set of studies, Me JA was added at the time of inoculation, but exposure was ended at defined intervals by removing from the plate the entire piece of agar where the MeJA had been applied (on the right in Fig. 1).

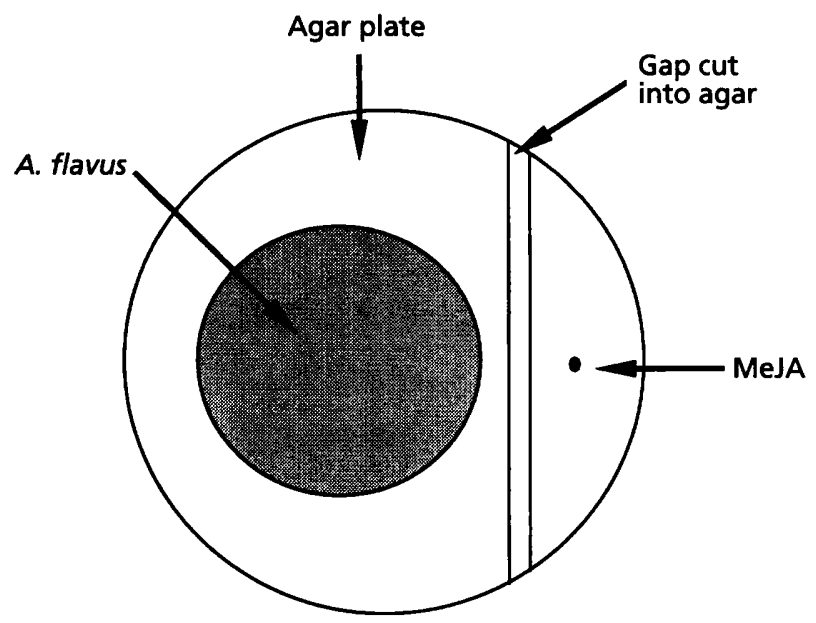

Fig. 1. Method of exposure of A. flavus cultures to airborne MeJA. A $3 \mathrm{~mm}( \pm 1 \mathrm{~mm})$ strip of agar was removed from the plate with a sterile scalpel blade, baring the bottom surface of the plate, and leaving a gap in the medium which extended the entire length (off-centre) of the plate, as shown. A. flavus spores were inoculated on the wider section of agar, and MeJA $(0.2 \mu l)$ was applied on the narrower section opposite the gap, as illustrated.

To examine the effect of MeJA treatment on aflatoxin levels in stored nuts, freshly field-harvested unshelled pistachio nuts were partially dried (15\% residual water by weight) and kept in containers at $25{ }^{\circ} \mathrm{C}$, allowing growth of the natural microflora. Each container (30.9 1) held $55 \mathrm{~kg}$ pistachios, incubated in either the presence or absence of MeJA vapour. For exposure to the vapour, the pistachios were equilibrated with a $100 \mu \mathrm{l}$ pool of MeJA in a watchglass placed above the nuts. The containers were covered but not airtight. After $13 \mathrm{~d}, 20$ randomly chosen samples of 100 nuts from both treatments were removed from the containers for aflatoxin assays. At this time, the pistachios from both containers showed extensive fungal contamination (mostly $A$, niger, with lesser contamination by A. flavus).

Aflatoxin and other biochemical assays. For assay of aflatoxins in pistachio nuts, each 100-nut sample was weighed and ground in a 11 blender. One-tenth of the ground material (about $15 \mathrm{~g}$ ) from each sample was removed for aflatoxin analysis. This material was blended for $1 \mathrm{~min}$ with $3 \mathrm{~g} \mathrm{NaCl}$ in $75 \mathrm{ml}$ methanol/water $(60: 40, \mathrm{v} / \mathrm{v})$ and then centrifuged at $5000 \mathrm{~g}$ for $10 \mathrm{~min}$. A $5 \mathrm{ml}$ aliquot of the supernatant was serially diluted to a final dilution of 1:5000. Ten millilitres of this dilution was applied to an aflatest-P (VICAM) immunoaffinity column. The column was washed with 2 column vols water, and aflatoxins were then eluted with $2 \mathrm{ml}$ acetonitrile. The acetonitrile was taken to dryness at $40^{\circ} \mathrm{C}$ with $\mathrm{N}_{2}$. The residue was derivatized with $200 \mu \mathrm{l}$ each of trifluoroacetic acid and hexane for $10 \mathrm{~min}$ at room temperature, then again taken to dryness at $40^{\circ} \mathrm{C}$ with $\mathrm{N}_{2}$, and dissolved in $200 \mu \mathrm{l}$ acetonitrile/water $(1: 9, \mathrm{v} / \mathrm{v})$. The extract was syringe-filtered and analysed by reverse-phase HPLC (Rodriguez \& Mahoney, 1994).

For in vitro assays, aflatoxins were extracted from cultures (entire plate, containing fungus and agar) and quantified by HPLC as detailed by Rodriguez \& Mahoney (1994).

The aflatoxin precursors sterigmatocystin and $O$-methylsterigmatocystin, and the $A$. flavus secondary metabolite cyclopiazonic acid, were identified by comparison of migration and 
staining properties following TLC of extracts and standards (Rodriguez \& Mahoney, 1994).

Fatty acid composition of $A$. flavus was determined on liquidgrown cultures and on spores by lipid extraction and GC analysis of methyl esters of total fatty acids (Goodrich-Tanrikulu et al., 1994).

\section{RESULTS}

\section{Effects of jasmonates on growth and aflatoxin production of $A$. flavus in vitro}

The aflatoxin which is usually produced at the highest levels by toxigenic strains of $A$. flavus is aflatoxin $\mathrm{B}_{1}$, which is also the most potent aflatoxin (Payne, 1992). We first detected aflatoxin $\mathrm{B}_{1} 3 \mathrm{~d}$ after $A$. flavus inoculation onto VMN. Aflatoxin $\mathrm{B}_{1}$ production peaked at about $9 \mathrm{~d}$, falling off gradually thereafter (Fig. 2). Aflatoxin $B_{2}$ was present only at trace levels. In cultures treated with $10^{-4}$ $\mathrm{M} \mathrm{MeJA}$, similar kinetics of aflatoxin production occurred, but the production of aflatoxin was inhibited approximately $80 \%$ throughout the time course (Fig. 2). Colony growth was essentially unaffected, aside from a slightly smaller ( $95 \%$ of untreated control) colony diameter through day 7 (data not shown). At day 8 , the mean colony diameter was $45 \mathrm{~mm}$ for both cultures.

Germination of spores was slightly delayed by $\mathrm{MeJA}$, enough to account for the slight early difference in colony size: on average, $50 \%$ of untreated spores had germ tubes $9 \cdot 1 \mathrm{~h}$ after inoculation. MeJA-treated spores germinated at the same rate, but with a $1.6 \mathrm{~h}$ delay. Spore viability was not affected.

Consequently, MeJA inhibited aflatoxin production per se, and unlike many other inhibitors (Zaika \& Buchanan, 1987; Rodriguez \& Mahoney, 1994), with no apparent

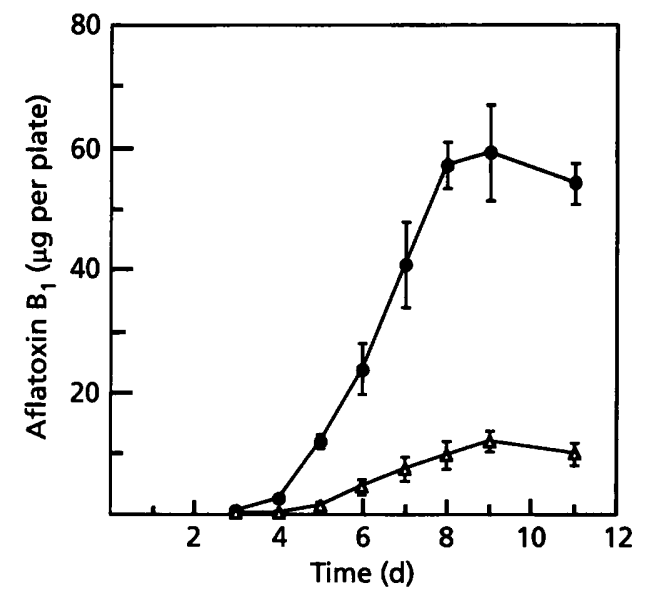

Fig. 2. Effect of MeJA on aflatoxin production over time by $\boldsymbol{A}$. flavus. $\triangle$, MeJA-treated cultures; 0 , untreated control cultures. Results presented are means \pm SE of three or four experiments using triplicate plates. Spores of $A$. flavus strain 42-12 were inoculated onto the centre of VMN agar with or without $10^{-4}$ $M \mathrm{MeJA}$, and harvested for aflatoxin analysis at the specified times. Aflatoxin $B_{1}$ was quantified by HPLC.

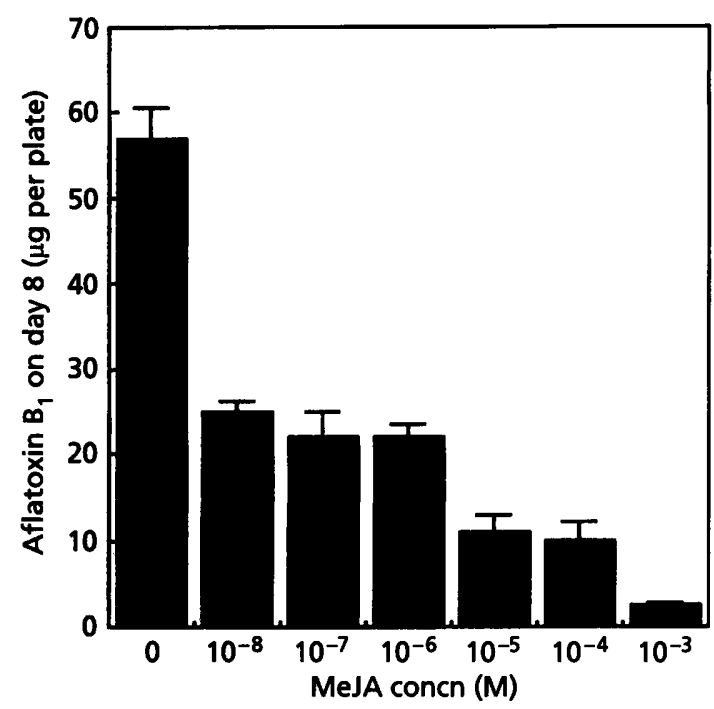

Fig. 3. Effect of various concentrations of MeJA in minimal medium on the amount of aflatoxin $B_{1}$ produced (culture plus medium) $\mathbf{8 d}$ after inoculation. Results are means $\pm \mathrm{SE}$ of two experiments using triplicate plates inoculated and assayed as described in Fig. 2 , except that the MeJA concentration in the medium varied as indicated.

effect on mycelial growth or colony appearance. In contrast with the lack of observed effect on growth, the yellow mycelial pigmentation found in untreated cultures was absent at all concentrations of MeJA tested. Additionally, droplets of undiluted MeJA applied near colonies caused local growth avoidance, and spores in the vicinity of this zone produced a yellow-orange rather than green pigment.

Aflatoxin production was inhibited at all levels of MeJA tested, ranging from $10^{-3}$ to $10^{-8} \mathrm{M}$ (Fig. 3); at the highest concentration tested, MeJA treatment reduced aflatoxin production by $96 \% 8 \mathrm{~d}$ after inoculation.

MeJA also inhibited aflatoxin production by $A$. flavus growing on other media, such as CYA medium, and on pistachios in storage (described later). Aflatoxin production in cultures treated with $10^{-4} \mathrm{M} \mathrm{MeJA}$ was $52 \%$ that of untreated cultures (which contained a mean of $31 \mu \mathrm{g}$ aflatoxin $\mathrm{B}_{1}$ per plate at day 8 ) in experiments using CYA, a complex medium (Rodriguez \& Mahoney, 1994), compared to $17 \%$ of untreated cultures at day 8 on VMN, a defined minimal medium (Fig. 2). Substrate composition thus influences the degree of inhibition.

Each of the responses which we observed was more pronounced for MeJA than for JA: JA at equivalent concentrations was less effective at inhibiting aflatoxin production ( $35 \%$ inhibition at $10^{-4} \mathrm{M}$ in VMN), delaying spore germination, and suppressing mycelial pigmentation. The lower effectiveness of JA compared to Me JA could be due to the unesterified compound (JA) having either a lower biochemical specificity, or an ionic character, which would be expected to lower membrane permeability. 

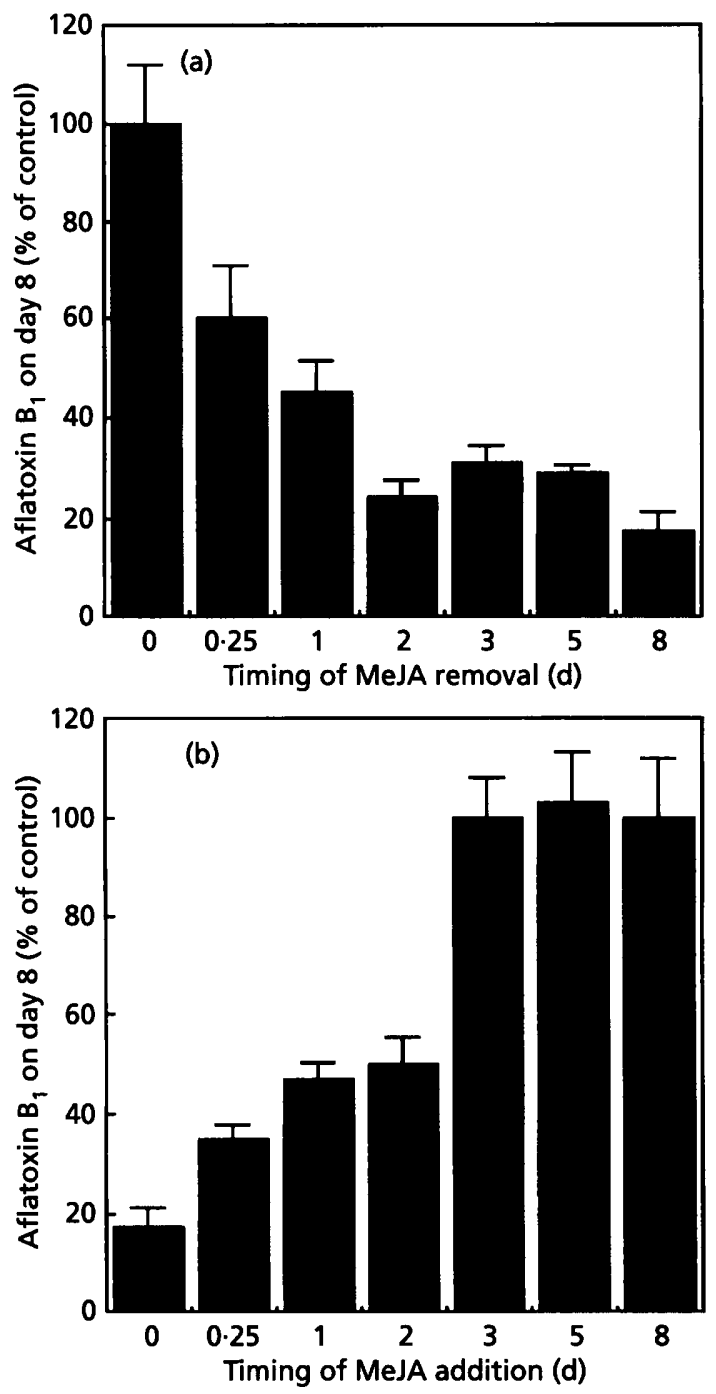

Fig. 4. Effect of duration of exposure to airborne MeJA on aflatoxin production by $A$. flavus. Data are means \pm SE of two experiments using triplicate plates, expressed relative to untreated controls $\left(31 \mu \mathrm{g}\right.$ aflatoxin $B_{1}$ per plate in these experiments). A. flavus 42-12 spores were inoculated onto VMN agar on day 0 and were exposed to MeJA as described in Fig. 1 and Methods. (a) Production of aflatoxin $B_{1}$ on day 8 when exposure to MeJA began at the time of inoculation and ended at specified times after inoculation. (b) Production of aflatoxin $B_{1}$ on day 8 when exposure to MeJA began at specified times after inoculation, and ended at time of harvest on day 8.

MeJA treatment apparently inhibited the biosynthesis of aflatoxin $B_{1}$. The immediate biochemical precursors of aflatoxin $\mathrm{B}_{1}$, sterigmatocystin and $O$-methylsterigmatocystin (Payne, 1992), did not accumulate in cultures treated with $10^{-4} \mathrm{M}$ MeJA (TLC plate not shown). The inhibition is thus likely to be prior to the formation of these compounds in the aflatoxin biosynthetic pathway. Inhibition may occur prior to the formation of the coloured anthroquinone intermediates early in the pathway, although the identity of the pigment(s) absent in mycelia of MeJA-treated cultures is not known. Levels of another secondary metabolite produced by $A$. flavus, cyclopiazonic acid (Luk et al., 1977), were unaffected by treatment (TLC plate not shown), demonstrating that the inhibition of aflatoxin production could be a specific effect, rather than a general one on secondary metabolism.

\section{Effect of exposure to gaseous MeJA on aflatoxin production in vitro and on pistachio nuts}

Inhibition of aflatoxin production occurred whether colonies were exposed to $\mathrm{MeJA}$ incorporated into the solid medium, or exposed to it in the gaseous phase. In experiments where MeJA was applied opposite a gap in the agar medium from the colony, it was similarly effective at reducing aflatoxin levels (Fig. 4). Thus, a source of $\mathrm{MeJA}$ need not necessarily be at the site of fungal growth to inhibit aflatoxin production.

A limited exposure to airborne MeJA was sufficient to reduce the production of aflatoxin. Exposure for only $6 \mathrm{~h}$ after inoculation was sufficient to inhibit total aflatoxin production by $39 \%$ at day 8 (Fig. 4a). This is remarkable, because $6 \mathrm{~h}$ after inoculation is prior to germ tube emergence (see earlier): exposure of single-celled germinating spores to $\mathrm{MeJA}$ inhibited aflatoxin production of the large $(45 \mathrm{~mm})$ multicellular colonies developing from them. Exposure for either 2 or $8 \mathrm{~d}$ after inoculation resulted in maximum inhibition, despite the increase in colony diameter from 20 to $45 \mathrm{~mm}$ between 2 and $8 \mathrm{~d}$ (an increase in area of nearly threefold). These results suggest that new fungal mycelia growing after MeJA exposure has ended retain $\mathrm{MeJA}$-induced inhibition of aflatoxin production. Complicating this interpretation is the observation that part of the total inhibition at $8 \mathrm{~d}$ was potentially due to residual MeJA vapour in the Petri dishes after removal of the source of MeJA. Twelve percent inhibition was found in plates inoculated after pretreatment with MeJA (plates incubated for $6 \mathrm{~h}$ with $\mathrm{MeJA}$ as illustrated in Fig. 1, followed by removal of source, then inoculation). Thus, the net inhibition after a limited-duration exposure appears to be due to a combination of continuing inhibition after removal of MeJA, and traces of residual MeJA, which are less effective, but still potent.

Exposure to MeJA was required early, when conditions are favourable for spore germination and growth, to achieve inhibition of aflatoxin biosynthesis in vitro. Airborne MeJA applied $6 \mathrm{~h}$ after inoculation onto a favourable growth medium was partially effective at reducing later aflatoxin production, but application $3 \mathrm{~d}$ or more after inoculation was completely ineffective (Fig. 4b). Pretreatment of spores with MeJA prior to inoculation was also ineffective (data not shown). The inhibition of aflatoxin production thus requires exposure within an early phase of growth.

We also tested the effectiveness of airborne MeJA treatment on field strains of aflatoxin-producing fungi growing on a natural substrate, pistachio nuts. The natural microflora was allowed to grow on incompletely dried pistachios (15\% residual moisture) for $13 \mathrm{~d}$ in the 

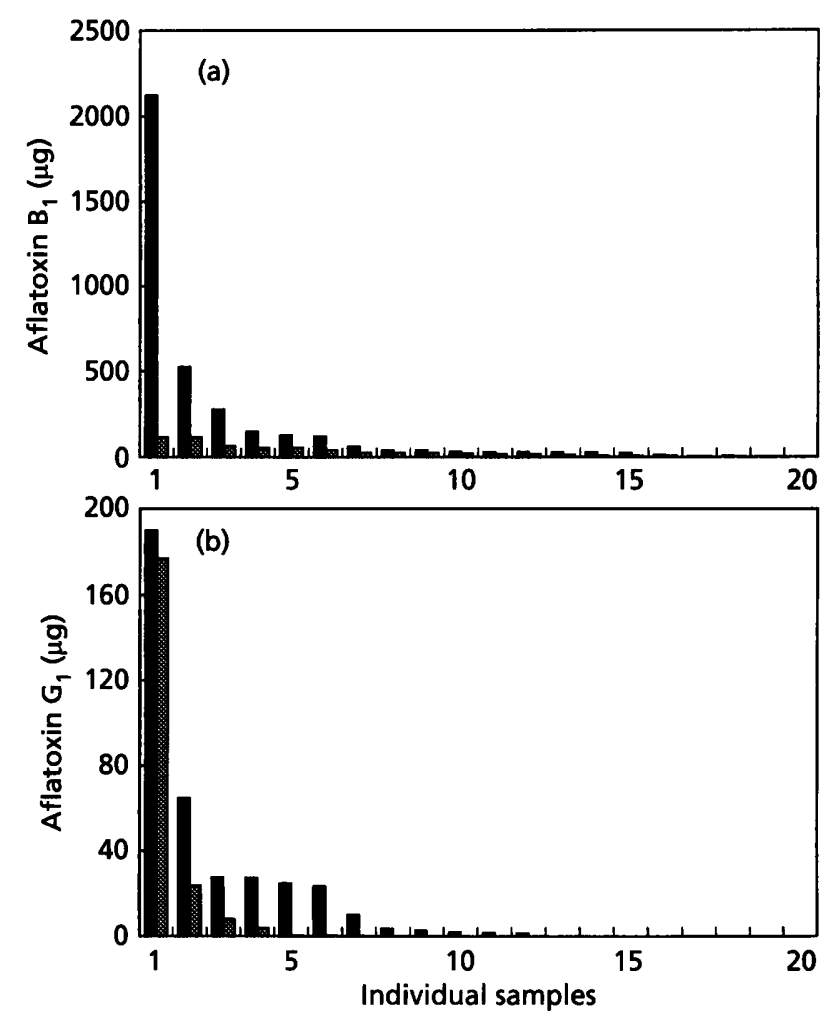

Fig. 5. Aflatoxin content of pistachio nuts incubated with airborne MeJA. Partially dried pistachios containing natural microflora were stored for $13 \mathrm{~d}$ in the presence or absence of MeJA as described in Methods. For both treatments, 20 individual samples (each containing 100 nuts) were assayed for aflatoxin content as described in Methods. Vertical bars show aflatoxin levels for each of the 20 samples, which have been ranked from the highest to lowest levels. Solid bars, untreated nuts; shaded bars, MeJA-treated nuts. (a) Profile of aflatoxin $B_{1}$ levels detected per 100-nut sample. (b) Profile of aflatoxin $G_{1}$ levels detected per 100-nut sample; note difference in scale from (a).

presence or absence of MeJA vapour, prior to assaying aflatoxin levels. At this time, no difference in extent of fungal contamination was noted between treatments, and some nuts in both treatments were visibly infected by $A$. flavus. Both aflatoxins $B_{1}$ and $G_{1}$ were detected in random samples of nuts, although the maximum levels of aflatoxin $\mathrm{B}_{1}$ formed were higher than those of $\mathrm{G}_{1}$ (Fig. 5). Aflatoxin $\mathrm{B}_{1}$ is produced by both $A$. flavus and $A$. parasiticus, whereas aflatoxin $\mathrm{G}_{1}$ is produced by $A$. parasiticus (Payne, 1992). The natural microflora thus included both $A$. flavus and $A$. parasiticus.

The aflatoxin levels assayed in the nuts showed characteristic variability, with skewed (non-normal) distributions (Fig. 5). MeJA-treated nuts had lower levels of both aflatoxins $B_{1}$ (Fig. 5a) and $G_{1}$ (Fig. 5b) than untreated nuts; the reduction in sampled aflatoxin levels is significant $(99 \%$, sign test). Based on these samples, MeJA treatment may be more effective against aflatoxin $\mathrm{B}_{1}$ production than against $G_{1}$ production (compare Fig. 5a, b). Six out of 20 of the untreated samples exceeded the highest levels of aflatoxin $B_{1}$ formed in the presence of
MeJA (Fig. 5a); the MeJA-treated nuts also had more samples with trace or no detectable aflatoxin (Fig. 5). Overall, nuts incubated with MeJA vapour had mean aflatoxin $B_{1}$ levels $17 \%$ and aflatoxin $G_{1}$ levels $56 \%$ of those of untreated nuts. Total aflatoxins in the MeJAtreated nuts were thus $20 \%$ of untreated nuts $(799 \mu \mathrm{g}$ and $3997 \mu \mathrm{g}$, respectively). These data demonstrate a broad effectiveness of MeJA on inhibition of production of aflatoxins by field strains of $A$. flavus and $A$. parasiticus growing on plant material, at least in pistachios.

\section{DISCUSSION}

\section{Jasmonate production by fungi}

$\mathrm{MeJA}$ inhibited aflatoxin production at levels lower than those reported for other plant-derived compounds inhibiting aflatoxin biosynthesis (reviewed by Zaika \& Buchanan, 1987), including compounds likewise derived from fatty acid hydroperoxides (Fabbri et al., 1983; Zeringue \& McCormick, 1990; Zeringue \& Bhatnagar, 1994). For example, in the presence of $2 \mu \mathrm{M}$ hexanal, $A$. parasiticus shows radial growth $76 \%$ and aflatoxin $B_{1}$ production $56 \%$ that of untreated cultures (Zeringue \& Bhatnagar, 1994); lower levels may be effective, but were apparently not tested.

MeJA was effective at reducing aflatoxin $B_{1}$ production at concentrations at least as low as $10^{-8} \mathrm{M}$ (Fig. 3). Because of its effectiveness at submicromolar concentrations, and its residual effectiveness after exposure has ended (Fig. $4 a)$, it is unlikely to directly affect enzymic activity. Instead, this high potency, and the observation of additional effects on pigmentation and spore germination, suggest a regulatory response mediated by a receptor. The observed level of effectiveness is probably an underestimate for two reasons. First, commercial preparations of jasmonates contain two enantiomers, only one of which may be physiologically active in plants (Vick, 1993). Second, since it is volatile, some MeJA originally in the medium will be lost over time. A high specificity for $\mathrm{MeJA}$ is also indicated by the lesser effectiveness of the structurally related compound JA, as noted above.

One of the $A$. flavus responses triggered by this putative receptor is an apparent inhibition of the biosynthesis or (indirectly) the activity of an enzyme or enzymes in the aflatoxin biosynthetic pathway. This and the other responses we observed to MeJA have parallels in plant systems, in which MeJA acts as a growth regulator: jasmonates affect the production of many secondary metabolites (Parthier, 1991; Sembdner \& Parthier, 1993; Aerts et al., 1994) and pigments, and delay pollen germination (Parthier, 1991; Sembdner \& Parthier, 1993). The range of effective concentrations and the kinetics of $\mathrm{MeJA}$ responses in plants are remarkably similar to those observed for $A$. flavus. The similarity of the $A$. flavus responses to $\mathrm{MeJA}$, and their pleiotropic nature, suggests that MeJA may act as a growth regulator in this fungus. At least two species of fungi, both plant pathogens, produce jasmonates (Aldridge et al., 1971; Miersch et al., 1992), and many others produce the fatty acid precursor $\alpha$ linolenate (Staswick, 1992). 
We do not know whether $A$. flavus produces jasmonates endogenously; $\alpha$-linolenate has not been detected in lipid extracts of $A$. flavus (Fernando \& Bean, 1986). We also could not detect this fatty acid in $A$. flavus extracts under our in vitro growth conditions. However, assuming this fungus, like other fungi including $A$. niger (Chattopadhyay et al., 1985), incorporates fatty acids from its substrate, these fatty acids are capable of entering the lipoxygenase pathway, if present. Thus, $A$. flavus may be capable of forming jasmonates under conditions where $\alpha$ linolenate is supplied in its substrate.

Inhibition of fungal spore germination by jasmonates has been previously reported for two other fungi, both plant pathogens (Neto et al., 1991; Cohen et al., 1993). In addition, growth of other structures of plant symbiotic or pathogenic fungi is inhibited (Gogala, 1991; Neto et al., 1991; Schweizer et al., 1993) or stimulated (Cohen et al., 1993) by jasmonates. Other metabolites of the polyunsaturated fatty acids $\alpha$-linolenate and $\alpha$-linoleate also affect fungal growth (Zeringue \& McCormick, 1990; Gogala, 1991; Neto et al., 1991). Metabolites of arachidonic acid, a polyunsaturated fatty acid synthesized by animals and many fungi (but not found in $A$. flavus or higher plants), can elicit plant defence responses (Choi et al., 1994), and are important endogenous regulatory molecules in animals and some fungi (Sembdner \& Parthier, 1993; Choi et al., 1994). Thus, the potential importance of jasmonates and structurally related compounds as fungal regulatory molecules is significant.

\section{MeJA and aflatoxin control}

Fatty acid hydroperoxides stimulate aflatoxin production (Fabbri et al., 1983; Fanelli \& Fabbri, 1989), whereas several of their metabolites, including various aldehydes (Zeringue \& McCormick, 1990; Zeringue, 1991) and jasmonates (noted here), generally inhibit aflatoxin production. The net result of simultaneous exposure of $A$. flavus cultures to fatty acid hydroperoxides and their metabolites is not known. Effects in different systems may depend on many factors, such as the fatty acid substrate, lipoxygenase specificity, and the levels of various other enzymes involved in hydroperoxide metabolism.

Much interest has been focused on factors providing crop resistance to aflatoxin contamination. Soybeans, unlike many other oilseeds, are resistant to aflatoxin contamination (Doehlert et al., 1993). Soybean oil is high in $\alpha$ linoleic and $\alpha$-linolenic acids, and soybeans contain an active lipoxygenase capable of forming the 13(S)-hydroperoxides from these fatty acids; the lipoxygenase pathway has been implicated as one important factor in the resistance of soybeans (Doehlert et al., 1993). Hexanals and hexenals, which have antifungal properties, are suspected to be involved, although detected levels of hexenals derived from $\alpha$-linolenate are lower than the levels anticipated to inhibit aflatoxin production (Doehlert et al., 1993). This suggests that resistance could be due at least in part to the conversion of 13hydroperoxylinolenate by either the fungus or soybeans to alternative products, e.g. jasmonates.
MeJA could be a useful tool for elucidating the control of the aflatoxin biosynthetic pathway. Our observation that the timing of Me JA exposure was critical to its effectiveness in A. flavus (Fig. 4) is consistent with studies of the suppression of aflatoxin biosynthetic capability by protein and RNA synthesis inhibitors in $A$. parasiticus (Buchanan et al., 1987; Cleveland \& Bhatnagar, 1989). Both types of studies have demonstrated that commitment to aflatoxin production occurs early in the growth of these fungi.

The effectiveness of Me JA further suggests a potential use in control of the production of aflatoxin in susceptible commodities. Since Me JA is a plant growth regulator, use in the field for aflatoxin control is not advisable. However, aflatoxin contamination can also occur postharvest, if the crop is not properly dried and stored (Payne, 1992). Based on our observations with pistachios, exposure to MeJA is likely to reduce levels of aflatoxin contamination which would otherwise occur in cases where the crop has been incompletely dried (Fig. 5), or accidentally or deliberately wetted. Our in vitro studies suggest that a brief exposure (i.e. $2 \mathrm{~d}$; Fig. $4 \mathrm{a}$ ) at this stage may be sufficient to minimize contamination. Including a source of $\mathrm{MeJA}$ in crop storage areas could be an effective, simple and safe means of reducing postharvest aflatoxin production.

\section{ACKNOWLEDGEMENTS}

We thank Bedoukian Research Incorporated, Danbury, CT, USA for their gift of methyl jasmonate.

\section{REFERENCES}

Aerts, R. J., Gisi, D., De Carolis, E., De Luca, V. \& Baumann, T. W. (1994). Methyl jasmonate vapor increases the developmentally controlled synthesis of alkaloids in Catharanthus and Cinchona seedlings. Plant J 5, 635-643.

Aldridge, D. C., Galt, S., Giles, D. \& Turner, W. B. (1971). Metabolites of Lasiodiplodia theobromae. J Chem Soc Sect C 1971, 1623-1627.

Buchanan, R. L., Jones, S. V., Gerasimowicz, W. V. \& Zaika, L. L. (1987). Regulation of aflatoxin biosynthesis: assessment of the role of cellular energy status as a regulator of the induction of aflatoxin production. Appl Environ Microbiol 53, 1224-1231.

Chattopadhyay, P., Banerjee, S. K., Sen, K. \& Chakrabarti, P. (1985). An unsaturated fatty acid mutant of Aspergillus niger with partially defective $\Delta$ 9-desaturase. Can J Microbiol 31, 346-351.

Choi, D., Bostock, R. M., Avdiushko, S. \& Hildebrand, D. F. (1994). Lipid-derived signals that discriminate wound- and pathogenresponsive isoprenoid pathways in plants: methyl jasmonate and the fungal elicitor arachidonic acid induce different 3-hydroxy-3methylglutaryl-coenzyme $\mathrm{A}$ reductase genes and antimicrobial isoprenoids in Solanum tuberosum L. Proc Natl Acad Sci US A 91, 2329-2333.

Cleveland, T. E. \& Bhatnagar, D. (1989). Evidence for de novo synthesis of an aflatoxin pathway methyltransferase near the cessation of active growth and the onset of aflatoxin biosynthesis in Aspergillus parasiticus mycelia. Can J Microbiol 36, 1-5.

Cohen, Y., Gisi, U. \& Niderman, T. (1993). Local and systemic protection against Pbytophtbora infestans induced in potato and tomato plants by jasmonic acid and jasmonic methyl ester. Phytopatbology 83, 1054-1062.

Davis, R. H. \& deSerres, F. J. (1970). Genetic and microbiological 
research techniques for Neurospora crassa. Methods Enzymol 17A, 79-143.

Doehlert, D. C., Wicklow, D. T. \& Gardner, H. W. (1993). Evidence implicating the lipoxygenase pathway in providing resistance to soybeans against Aspergillus flavus. Phytopathology 83, 1473-1477.

Fabbri, A. A., Fanelli, C., Panfili, G., Passi, S. \& Fasella, P. (1983). Lipoperoxidation and aflatoxin biosynthesis by Aspergillus parasiticus and $A$. flavus. J Gen Microbiol 129, 3447-3452.

Fanelli, C. \& Fabbri, A. A. (1989). Relationship between lipids and aflatoxin biosynthesis. Mycopatbologia 107, 115-120.

Fernando, T. \& Bean, G. (1986). Effects of tricyclazole on growth, release of aflatoxin, and sterol and fatty acid content by Aspergillus isolates. Trans Br Mycol Soc 87, 445-449.

Gogala, N. (1991). Regulation of mycorrhizal infection by hormonal factors produced by hosts and fungi. Experientia 47, 331-340.

Goodrich-Tanrikulu, M., Stafford, A. E., Lin, J.-T., Makapugay, M. I., Fuller, G. \& McKeon, T. A. (1994). Fatty acid biosynthesis in novel ufa mutants of Neurospora crassa. Microbiology 140, 2683-2690.

Luk, K. C., Kobbe, B. \& Townsend, J. M. (1977). Production of cyclopiazonic acid by Aspergillus flavus Link. Appl Environ Microbiol 33, 211-212.

Merzlyak, M. N. (1990). Syndrome of lipid peroxidation in plants. In Plant Lipid Biochemistry, Structure and Utilization. Proceedings of the Ninth International Symposium on Plant Lipids, pp. 281-288. Edited by P. J. Quinn \& J. L. Harwood. London: Portland Press.

Miersch, O., Bruckner, B., Schmidt, J. \& Sembdner, G. (1992). Cyclopentane fatty acids from Gibberella fujikuroi. Pbytochemistry 31, 3835-3837.

Neto, G. C., Kono, Y., Hyakutake, H., Watanabe, M., Suzuki, Y. \& Sakurai, A. (1991). Isolation and identification of (-)-jasmonic acid from wild rice, Oryza officinalis, as an antifungal substance. Agric Biol Chem 55, 3097-3098.

Parthier, B. (1991). Jasmonates, new regulators of plant growth and development: many facts and few hypotheses on their actions. Bot Acta 104, 446-454.
Payne, G. A. (1992). Aflatoxin in maize. Crit Rev Plant Sci 10, 423-440.

Rodriguez, S. B. \& Mahoney, N. E. (1994). Inhibition of aflatoxin production by surfactants. Appl Environ Microbiol 60, 106-110.

Sanders, T. H., Cole, R. J., Blankenship, P. D. \& Dorner, J. W. (1993). Aflatoxin contamination of peanuts from plants drought stressed in pod or root zones. Peanut Sci 20, 5-8.

Schweizer, P., Gees, R. \& Mosinger, E. (1993). Effect of jasmonic acid on the interaction of barley (Hordeum vulgare L.) with the powdery mildew Erysiphe graminis f. sp. hordei. Plant Physiol 102, 503-511.

Sembdner, G. \& Parthier, B. (1993). The biochemistry and the physiological and molecular actions of jasmonates. Annu Rev Plant Pbysiol Plant Mol Biol 44, 569-589.

Staswick, P. E. (1992). Jasmonate, genes, and fragrant signals. Plant Physiol 99, 804-807.

Vick, B. A. (1993). Oxygenated fatty acids of the lipoxygenase pathway. In Lipid Metabolism in Plants, pp. 167-191. Edited by T. S. Moore, Jr. Boca Raton, FL: CRC Press.

Zaika, L. L. \& Buchanan, R. L. (1987). Review of compounds affecting the biosynthesis or bioregulation of aflatoxins. J Food Prot 50, 691-708.

Zeringue, H. J., Jr (1991). Effect of $\mathrm{C} 6$ to $\mathrm{C} 9$ alkenals on aflatoxin production in corn, cottonseed, and peanuts. Appl Environ Microbiol 57, 2433-2434.

Zeringue, H. J., Jr \& Bhatnagar, D. (1994). Effects of neem leaf volatiles on submerged cultures of aflatoxigenic Aspergillus parasiticus. Appl Environ Microbiol 60, 3543-3547.

Zeringue, H. J., Jr \& McCormick, S. P. (1990). Aflatoxin production in cultures of Aspergillus flavus incubated in atmospheres containing selected cotton leaf-derived volatiles. Toxicon 28, 445-448.

Received 12 May 1995; revised 25 July 1995; accepted 26 July 1995. 